Original Research

\title{
Distribution Characteristics of Selenium Nutrition on the Natural Habitat of Przewalski's Gazelle
}

\author{
Yongkuan Chi ${ }^{1,2}$, Bin Huo ${ }^{1}$, Xiaoyun Shen ${ }^{1,2,3 *}$ \\ ${ }^{1}$ State Engineering Technology Institute for Karst Desertification Control, Guizhou Normal University, Guiyang, China \\ ${ }^{2}$ School of Life Science and Engineering, Southwest University of Science and Technology, Mianyang, China \\ ${ }^{3}$ World Bank Poverty Alleviation Project Office in Guizhou, Southwest China, Guiyang, China
}

Received: 18 December 2018

Accepted: 24 February 2019

\begin{abstract}
Selenium is an essential mineral nutrient for animal growth. Previous studies have shown a serious imbalance in selenium distribution in the habitat of Przewalski's gazelle (Procapra przewalskii). In order to provide new ideas for wildlife conservation, we studied the distribution characteristics of selenium nutrition in the natural habitat of Przewalski's gazelle in the Qinghai Lake watershed. The results showed that the average selenium content of mixed pasture in the Hudong area was the lowest, and was seriously selenium deficient; the upper reaches of the Buha River showed subclinical selenium deficiency; and the average selenium content of mixed pasture in Bird Island Reserve was the highest. Among soil layers, contents of total and water-soluble selenium were highest in surface soil, and selenium content decreased gradually with soil depth. Among soil types, the total selenium content was highest in boggy soil and lowest in gray cinnamon soil; the water-soluble selenium content was highest in boggy soil and lowest in alpine scrub meadow soil. Among grassland types, selenium content was highest in mixed forage of Achnatherum splendens grassland and lowest in mixed forage of Artemisia frigida grassland. The selenium content in mixed forage showed obvious seasonality, being highest at the seedling stage and lowest at the growing stage. Among forage species, Clematis tangutica, Artemisia desertorum and Achnatherum splendens were the main high-selenium plants, with the selenium content the highest for Achnatherum splendens. Poa pratensis, Elymus nutans and Thermopsis lanceolata were the main selenium-deficient plants, and selenium content was lowest in Thermopsis lanceolata.
\end{abstract}

Keywords: distribution characteristics, habitat, Przewalski's gazelle, Qinghai Lake watershed, selenium nutrition

*e-mail: sfdxsxy@163.com 


\section{Introduction}

Qinghai Lake, the largest inland saltwater lake in China, is located in the northeast of the Qinghai-Tibet Plateau and lies $3196 \mathrm{~m}$ above sea level. The Qinghai Lake watershed has abundant precipitation, rain and warmth during the same period, annual sunshine duration of more than 3000 hours, annual mean temperature within $0.3-1.1^{\circ} \mathrm{C}$, annual precipitation of $370-412 \mathrm{~mm}$ and extensive pastures and rich grassland resources. Thus, it has unique natural conditions to develop grassland animal husbandry, and is also the natural habitat of Przewalski's gazelle [1-2].

Przewalski's gazelle is a unique and endangered animal in China, and is the smallest in mammal species in the region [2]. The selenium intake of Przewalski's gazelle is mainly from forage, and the selenium content in forage depends on the water-soluble selenium in soil [4], which is affected by total selenium in soil and physical and chemical properties of soil, especially $\mathrm{pH}$ and redox potential [4-5]. In alkaline and alkalescent soils, selenium mainly exists as selenate and is easily dissolved in water; in acid and subacid soils, selenium mainly exists as selenite, and easily binds to iron in soil to form insoluble complexes that are not conducive to plant absorption [5-6]. The Qinghai Lake watershed is alpine meadow, the root systems of forage in the surface layer of soil are intertwining, air permeability is poor, and this results in an anaerobic environment that is not conducive to selenium oxidation into selenate and so can affect the selenium content of plants [1-2]. Plant species is the main factor affecting the selenium content of forage, and a previous study showed that Achnatherum splendens was the most important selenium-rich plant in the Qinghai Lake watershed [2]. Selenium is not needed for plant growth and development, but the requirement of animals mainly comes from plants, so if the habitat is deficient in selenium, selenium deficiency often occurs in animals. Selenium is an essential mineral for animals and the active center element of glutathione peroxidase in the animal body. Glutathione peroxidase is the most important functional protein of selenium and catalyzes reducing glutathione to scavenge free radicals [7-8]. Selenium can also improve the nonspecific immune function of the body. When an organism is deficient in selenium, the proliferation and differentiation of lymphocytes are inhibited, the secretion of lymphoid factors is reduced, the ability of lymphocytes and natural killer cells to kill cancer cells is decreased, and the ability of phagocytes to phagocytose and kill pathogens is seriously affected, antibody synthesis is reduced and disease resistance of the organism decreases [9-10]. The main symptoms of selenium deficiency in animals are decreased production performance, growth retardation, decreased, emaciation, skeletal muscle necrosis, cell membrane degeneration, protein damage and mutation of DNA, which eventually leads to various diseases in the body and even aging and death. Severe selenium deficiency leads to individual death, population collapse and species extinction [9].

Previous studies found an obvious imbalance in selenium distribution in the Qinghai Lake watershed [1-2], and this deficiency in natural grassland seriously affects the development of grassland and animal husbandry. Some studies have shown that the selenium content in blood, liver, muscle and other tissues of grazing sheep in the Hudong area was significantly lower than the normal reference value. The main clinical symptoms include emaciation, diarrhea, loss of appetite, growth retardation and decreased fecundity, and the most serious case is sudden death. Because the pathological symptoms include degeneration and necrosis of skeletal muscle, myocardium and liver tissue, it is also called white myopathy [1]. Although there is a problem of selenium deficiency in grazing livestock, it is not known if selenium deficiency also occurs in Przewalski's gazelle. The purpose of this study was to study the distribution characteristics of selenium nutrition in the natural habitat of Przewalski's gazelle in the Qinghai Lake watershed, and to explore the effect of the distribution of selenium nutrition in the habitat on the dynamic growth and development of the Przewalski's gazelle population so as to provide new ideas for its protection.

\section{Materials and Methods}

\section{Study Sites}

The experimental area included the Hudong area, Bird Island Reserve and the upper reaches of the Buha River in the Qinghai Lake watershed. This area has an average elevation of 3036-3526 $\mathrm{m}$ and a semiarid alpine climate with a large temperature difference and a short frost-free period. There are different natural grassland types in the area, such as Achnatherum splendens, Iris lactea, Stipa penicillata, Artemisia desertorum, Stellera chamaejasme and Stipa glareosa. The dominant plants are Leymus secalinus, Agropyron cristatum, Carex mooscroftii, Artemisia frigida, Carex stenophylla, Clematis tangutica, Stipa purpurei, Poa pratensis, Elymus nutans, Orinus kokonorica, Artemisia anethifolia, Agropyron cristatum and Thermopsis lanceolata (Fig. 1).

\section{Study Methods \\ Collection of Soil Samples}

Soil samples were collected using $1 \times 1 \mathrm{~m}$ quadrats with an interval of $200 \mathrm{~m}$. The collection sites included Hudong, Bird Island Reserve and the upper reaches of the Buha. The collected soil types included carbonate alpine meadow, alpine scrub meadow, gray cinnamon and boggy soils; soil samples of surface soil, subsoil and substratum layers were collected respectively. Twenty 


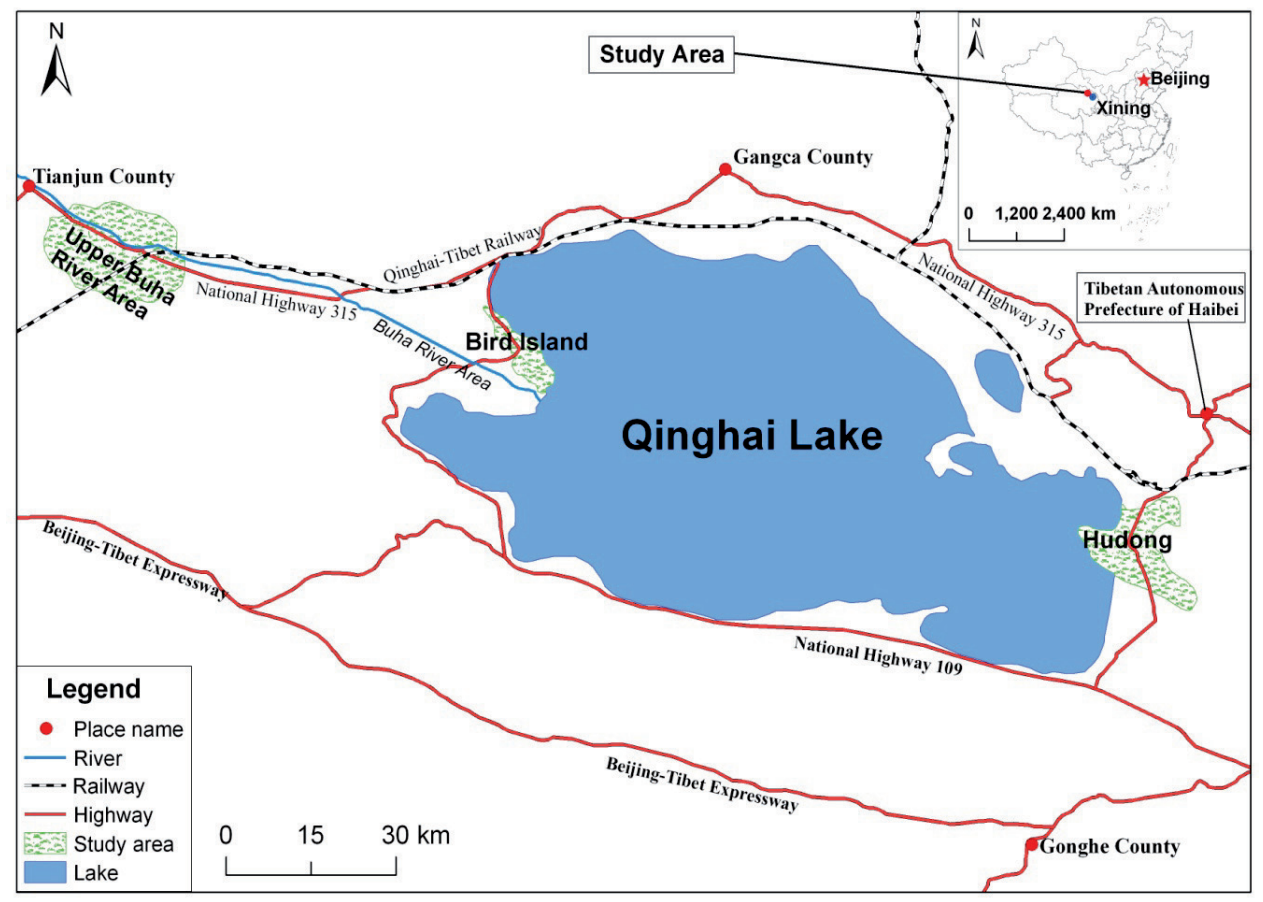

Fig. 1. Study area of the natural habitat of Przewalski's gazelle in the Tibetan Plateau in China.

samples were collected from Hudong Breeding Farm, which were divided into 20 sampling sites (among them, five samples were respectively collected from carbonate alpine meadow, alpine shrub meadow, gray cinnamon and boggy soils, with each sample of $200 \mathrm{~g}$ ). The number of samples and the collection methods in the upper reaches of the Buha and the Bird Island Reserve were the same as those in Hudong.

\section{Collection of Forage Samples}

The mixed forage samples were collected in natural grassland of Achnatherum splendens, Iris lactea and Stipa penicillata on 10 May 2017 (seedling stage), 10 July 2017 (growing period) and 10 September 2017 (withering period), and all sample sites were in Hudong Breeding Farm, Bird Island Reserve and the upper reaches of the Buha River. For each grassland type, five mixed forage samples (each of $100 \mathrm{~g}$ ) were randomly collected in the seedling, growing and withering stages of forage.

On 1 August 2017, 14 forages were collected from Hudong Breeding Farm, Bird Island Reserve and upper reaches of the Buha, including Achnatherum splendens, Stellera chamaejasme, Artemisia desertorum and Artemisia frigida. Five forage samples were collected from each forage variety, with each sample being $100 \mathrm{~g}$. The sample distribution, sample number and weight of forage sample were the same as for soil samples.

\section{Treatment of Soil and Forage Samples}

Soil samples were naturally dried at $20-25^{\circ} \mathrm{C}$ and crushed, then passed through a $2-\mathrm{mm}$ sieve and a $0.075-\mathrm{mm}$ sieve used to remove silver sand. In the experiment, $0.3 \mathrm{~g}$ of soil sample was taken and put into a tube, and $6 \mathrm{ml}$ of nitric acid $\left(\mathrm{HNO}_{3}\right)$ and $1 \mathrm{ml}$ of hydrogen peroxide $\left(\mathrm{H}_{2} \mathrm{O}_{2}\right)$ were added, then the tube was shaken well before standing for $10 \mathrm{~min}$. The solution was digested by microwave digestion and, after the solution had cooled, was transferred into a $100-\mathrm{ml}$ volumetric bottle where it was diluted to $100 \mathrm{ml}$ of total volume. The available selenium of soil was extracted using a $0.5 \mathrm{~mol} \cdot \mathrm{L}^{-1}(\mathrm{pH} 8.5)$ sodium bicarbonate solution.

The forage sample was naturally dried at $20-25^{\circ} \mathrm{C}$ and crushed, then passed through a $0.175-\mathrm{mm}$ sieve and bagged for use. Of each forage sample, $0.5 \mathrm{~g}$ was placed into a tube with $6 \mathrm{ml}$ of $\mathrm{HNO}_{3}$ and $1 \mathrm{ml}$ of $\mathrm{H}_{2} \mathrm{O}_{2}$, shaken well and stood for $10 \mathrm{~min}$. The solution was digested by microwave digestion and, after it had cooled, was transferred into a $100-\mathrm{ml}$ volumetric bottle, where it was diluted to $100 \mathrm{ml}$ of total volume.

\section{Analysis of Soil and Forage Samples}

Selenium contents in soil and forage were determined using an inductively coupled plasma atomic emission spectrometer (ICP-AES) with HK9600 type atomic emission spectroscopy (Huaketiancheng Co. Ltd, China). The operating conditions of the ICP-AES are shown in Table 1.

\section{Data Analysis}

The data were analyzed by SPSS 20.0 for Windows software, after the distribution of test data was checked for normal distribution, and the selenium content of forage in different species and periods was analyzed by 
Table 1. Operating conditions for determining selenium using an ICP-AES.

\begin{tabular}{|c|c|c|c|c|c|}
\hline Wave length & Radio-frequent Power & Carrier gas flow & Cryogenic gas & Integral time & Sampling time \\
\hline $520(\mathrm{~nm})$ & $1.2(\mathrm{~kW})$ & $0.4(\mathrm{~L} / \mathrm{min})$ & $15(\mathrm{~L} / \mathrm{min})$ & $5(\mathrm{~s})$ & $20(\mathrm{~s})$ \\
\hline
\end{tabular}

single-factor variance analysis. The experimental data are expressed as mean $(\bar{x}) \pm$ standard deviation $(\mathrm{S})$.

\section{Results}

\section{Distribution Characteristics of Total Selenium in Soil}

Of our three study areas, Hudong has serious selenium deficiency, the upper reaches of the Buha River area are selenium deficient and soil of the Bird Island Reserve meets the selenium needs of grazing livestock and wildlife. There were significant differences in total selenium content of surface soils among the three study areas (carbonate soil: $F_{2.12}=2.38, p=0.001$; alpine scrub soil: $F_{2,12}=3.31, p=0.002$; boggy soil: $F_{2,12}=3.47$, $p=0.003$; gray cinnamon soil: $F_{2,12}=3.22, p=0.005$ ). There were significant differences in total selenium content of subsoils among the three areas (carbonate soil: $F_{2,12}=3.57, p=0.003$; boggy soil: $F_{2,12}=3.74$, $p=0.004$; gray cinnamon soil: $F_{2,12}=3.48, p=0.008$ ). There were significant differences in total selenium content of substratum soils among the three areas (carbonate soil: $F_{2,12}=3.18, p=0.002$; boggy soil: $F_{2,12}=3.33, p=0.004$; gray cinnamon soil: $F_{2,12}=3.72$, $p=0.006$ ).

In the Hudong area, there were significant differences in total selenium content among carbonate, alpine scrub, boggy and gray cinnamon soils (surface soil of different soil types: $F_{3,16}=2.17, p=0.006$; subsoil: $F_{3,16}=2.37$, $p=0.005$; substratum soil: $F_{3,16}=2.11, p=0.004$ ). There were significant differences in total selenium content among surface soil, subsoil and substratum soil (carbonate soil: $F_{2,12}=3.12, p=0.008$; alpine scrub soil: $F_{2,12}=1.57, p=0.007$; boggy soil: $F_{2,12}=2.76, p=0.009$; gray cinnamon soil: $F_{2,12}=2.12, p=0.001$ ).

In the Bird Island Reserve, there were significant differences in total selenium content among carbonate, alpine scrub, boggy and gray cinnamon soils (surface soil of the four soil types: $F_{3,16}=3.16, p=0.005$; subsoil: $F_{3,16}=3.35, p=0.003$; substratum soil: $\left.F_{3,16}=3.17, p=0.007\right)$. There were significant differences in total selenium content among surface soil, subsoil and substratum soil (carbonate soil: $F_{2,12}=4.31, p=0.002$; alpine scrub soil: $F_{2,12}=3.17, p \stackrel{2}{=} 0.003$; boggy soil: $F_{2,12}=4.35, p=0.009$; gray cinnamon soil: $F_{2,12}=4.37$, $p=0.005)$.

In the upper reaches of the Buha River, there were significant differences in total selenium content among carbonate, alpine scrub, boggy and gray cinnamon soils (surface soil of the four soil types: $F_{3,16}=2.37, p=0.009$; subsoil: $F_{3,16}=2.23, p=0.006$; substratum soil: $\left.F_{3,16}=2.78, p=0.009\right)$. There were significant differences in total selenium content among surface soil, subsoil and substratum soil (carbonate soil: $F_{2,12}=3.33, p=0.005$; alpine scrub soil: $F_{2,12}=2.89$, $p=0.006$; boggy soil: $F_{2,12}=2.39, p=0.006$; gray cinnamon soil: $F_{2,12}=2.17, p=0.007$ ).

\section{Distribution Characteristics of Available Selenium in Soil}

There were significant differences in available selenium content of surface soil among the three study areas (carbonate soil: $F_{2,12}=3.17, p=0.002$; alpine scrub soil: $F_{2,12}=3.23, \stackrel{p}{p}=0.001$; boggy soil: $F_{2,12}=3.16, p=0.004$; gray cinnamon soil: $F_{2,12}=3.22$, $p=0.005)$. There were significant differences in available selenium content of subsoil among the three study areas (carbonate soil: $F_{2,12}=3.33, p=0.007$; boggy soil: $F_{2,12}=3.35, p=0.002$; gray cinnamon soil: $\left.F_{2,12}=3.17, p=0.004\right)$. There were significant differences in available selenium content of substratum soil among the three study areas (carbonate soil: $F_{2,12}=3.48$, $p=0.005$; boggy soil, $F_{2,12}=3.23, p=0.005$; gray cinnamon soil: $F_{2,12}=3.72, p=0.006$ ).

In the Hudong area, there were significant differences in available selenium content among carbonate, alpine scrub, boggy and gray cinnamon soils (surface soil of the four soil types: $F_{3,16}=5.36, p=0.010$; subsoil: $F_{3,16}=5.23, p=0.007$; substratum soil: $\mathrm{F}_{3,16}=5.11$, $\mathrm{P}=0.009)$. There were significant differences in available selenium content among surface soil, subsoil and substratum soil (carbonate soil: $F_{2,12}=4.32$, $p=0.002$; alpine scrub soil: $F_{2,12}=4.26, p=0.003$; boggy soil: $F_{2,12}=4.43, p=0.007$; gray cinnamon soil: $F_{2,12}=5.31, p \stackrel{2,12}{=} 0.006$ ).

In the Bird Island Reserve, there were significant differences in available selenium content among carbonate, alpine scrub, boggy and gray cinnamon soils (surface soil of the four soil types: $F_{3,16}=4.12$, $p=0.007$; subsoil: $F_{3,16}=4.32, p=0.004$; substratum soil: $\left.F_{3,16}=4.71, p=0.003\right)$. There were significant differences in available selenium content among surface soil, subsoil and substratum soil (carbonate soil: $F_{2,12}=3.37, p=0.005$; alpine scrub soil: $F_{2,12}=4.36$, $p=0.007$; boggy soil: $F_{2,12}=4.16, p=0.003$; gray cinnamon soil: $\left.F_{2,12}=4.21, p=0.007\right)$.

In the upper reaches of the Buha River, there were significant differences in available selenium content among carbonate, alpine scrub, boggy and gray cinnamon soils (surface soil of the four soil types: $F_{3,16}=6.17, p=0.001$; subsoil: $F_{3,16}=6.26, p=0.005$; substratum soil: $\left.F_{3,16}=6.38, p=0.002\right)$. There were significant differences in available selenium content 
Table 2. Total selenium content of soil in Przewalski's gazelle habitats $\left(\mu \mathrm{g} \cdot \mathrm{g}^{-1}\right)$.

\begin{tabular}{|c|c|c|c|c|}
\hline Regions & Soil types & Surface soil & Subsoil & Substratum soil \\
\hline \multirow{4}{*}{ Hudong area } & Carbonate soil & $0.0213 \pm 0.0035$ & $0.0165 \pm 0.0015$ & $0.0104 \pm 0.0013$ \\
\cline { 2 - 5 } & Alpine scrub soil & $0.0255 \pm 0.0041$ & $0.0187 \pm 0.0025$ & $0.0146 \pm 0.0014$ \\
\cline { 2 - 5 } & Boggy soil & $0.0916 \pm 0.0075$ & $0.0735 \pm 0.0023$ & $0.0515 \pm 0.0031$ \\
\cline { 2 - 5 } & Gray cinnamon soil & $0.0171 \pm 0.0011$ & $0.0135 \pm 0.0012$ & $0.0081 \pm 0.0017$ \\
\hline \multirow{4}{*}{\begin{tabular}{c} 
Bird Island Reserve \\
\cline { 2 - 5 }
\end{tabular}} & Carbonate soil & $0.0625 \pm 0.0031$ & $0.0455 \pm 0.0027$ & $0.0313 \pm 0.0031$ \\
\cline { 2 - 5 } & Alpine scrub soil & $0.0865 \pm 0.0046$ & $0.0637 \pm 0.0033$ & $0.0476 \pm 0.0032$ \\
\cline { 2 - 5 } & Gray cinnamon soil & $0.0411 \pm 0.0013$ & $0.0387 \pm 0.0022$ & $0.0333 \pm 0.0028$ \\
\hline \multirow{3}{*}{$\begin{array}{c}\text { Upper reaches } \\
\text { of Buha River }\end{array}$} & Carbonate soil & $0.0315 \pm 0.0035$ & $0.0265 \pm 0.0015$ & $0.0314 \pm 0.0013$ \\
\cline { 2 - 5 } & Alpine scrub soil & $0.0425 \pm 0.0041$ & $0.0327 \pm 0.0025$ & $0.0256 \pm 0.0014$ \\
\cline { 2 - 5 } & Boggy soil & $0.0996 \pm 0.0075$ & $0.0795 \pm 0.0023$ & $0.0695 \pm 0.0031$ \\
\cline { 2 - 5 } & Gray cinnamon soil & $0.0276 \pm 0.0011$ & $0.0205 \pm 0.0012$ & $0.0211 \pm 0.0017$ \\
\hline
\end{tabular}

among surface soil, subsoil and substratum soil (carbonate soil: $F_{2.12}=5.31, p=0.001$; alpine scrub soil: $F_{2,12}=5.79, p=0.002$; boggy soil: $F_{2,12}=5.37, p=0.003$; gray cinnamon soil: $F_{2,12}=5.12, p=0.002$ ).

Distribution Characteristics of Selenium in the Mixed Forage of Natural Grasslands

In the Hudong area, the selenium distribution in mixed forage significantly differed among grassland types. There were significant differences in selenium content of Stellera chamaejasme grassland, Artemisia desertorum scrub, Artemisia frigida grassland, Iris lactea grassland, Achnatherum splendens grassland, Stipa penicillata grassland, Stipa glareosa grassland and Stipa purpurei grassland (seedling stage: $F_{7,32}=3.15$, $p=0.007$; growing period: $F_{7,32}=3.27, p=0.003$; withering period: $F_{7,32}=3.21, p=0.002$ ). The seasonal dynamics of selenium content in the mixed forage were obvious (Stellera chamaejasme: $F_{2,12}=3.17, p=0.007$; Artemisia desertorum: $F_{2,12}=4.21, p=0.009$; Artemisia frigida: $F_{2,12}=3.15, p=0.002$; Iris lactea: $F_{2,12}=1.57$, $p=0.003$; Achnatherum splendens: $F_{2,12}=5.23$, $p=0.003$; Stipa penicillata: $F_{2,12}=2.16, p=0.004$; Stipa glareosa: $F_{2,12}=1.12, p=0.001$; Stipa purpurei: $F_{2,12}=2.79, p=0.010$ ).

In the Bird Island Reserve, the selenium distribution in mixed forage significantly differed among grassland types. There were significant differences in selenium content of Stellera chamaejasme grassland, Artemisia desertorum scrub, Artemisia frigida grassland, Iris lactea grassland, Achnatherum splendens grassland, Stipa penicillata grassland, Stipa glareosa grassland and Stipa purpurei grassland (seedling stage: $F_{7,32}=3.78$, $p=0.003$; growing period: $F_{7,32}=4.21, p=0.007$; withering period: $\left.F_{7,32}=5.35, p=0.007\right)$. The seasonal dynamics of selenium content in the mixed forage were obvious (Stellera chamaejasme: $F_{2,12}=4.72, p=0.007$; Artemisia desertorum: $F_{2,12}=3.21, p=0.009$; Artemisia frigida: $F_{2,12}=4.11, p=0.004$; Iris lactea: $F_{2,12}=5.31$, $p=0.006 ;$ Achnatherum splendens: $F_{2,12}=5.24$, $p=0.007$; Stipa penicillata: $F_{2,12}=5.32, p=0.007$; Stipa glareosa: $F_{2,12}=5.33, p=0.001$; Stipa purpurei: $\left.F_{2,12}=6.19, p=0.008\right)$.

In the upper reaches of the Buha River, selenium distribution in mixed forage significantly differed among grassland types. There were significant differences in selenium content of Stellera chamaejasme grassland, Artemisia desertorum scrub, Artemisia frigida grassland, Iris lactea grassland, Achnatherum splendens grassland, Stipa penicillata grassland, Stipa glareosa grassland and Stipa purpurei grassland (seedling stage: $F_{7,32}=5.25, p=0.005$; growing period: $F_{7,32}=4.28$, $p=0.008$; withering period: $\left.F_{7,32}=6.21, p=0.004\right)$. The seasonal dynamics of selenium content in the mixed forage were obvious (Stellera chamaejasme: $F_{2,12}=4.23$, $p=0.007$; Artemisia desertorum: $F_{2,12}=3.17, p=0.009$; Artemisia frigida: $F_{2,12}=5.21, p=0.003$; Iris lactea: $F_{2,12}=2.35, p=0.006 ;$ Achnatherum splendens: $F_{2,12}=7.13, p=0.005$; Stipa penicillata: $F_{2,12}=3.16$, $p=0.007$; Stipa glareosa: $F_{2,12}=5.12, p=0.008$; Stipa purpurei: $\left.F_{2,12}=3.73, p=0.004\right)$.

\section{Distribution Characteristics of Selenium in Different Forage Varieties}

In the Hudong area, there were significant differences in selenium content among forage varieties. There were very significant differences in selenium content among Artemisia frigida, Artemisia desertorum, Stellera chamaejasme, Achnatherum splendens, Iris lactea, Carex stenophylla, Stipa purpurei, Agropyron cristatum, Poa pratensis, Elymus nutans, Orinus kokonorica, Thermopsis lanceolata, Stipa penicillata, 
Table 3. Available selenium content of soil in Przewalski's gazelle habitats $\left(\mu \mathrm{g} \cdot \mathrm{g}^{-1}\right)$.

\begin{tabular}{|c|c|c|c|c|}
\hline Regions & Soil types & Surface soil & Subsurface soil & Substratum soil \\
\hline \multirow{4}{*}{ Hudong area } & Carbonate soil & $1.615 \pm 0.321$ & $1.235 \pm 0.176$ & $0.815 \pm 0.121$ \\
\cline { 2 - 5 } & Alpine scrub soil & $1.995 \pm 0.317$ & $1.495 \pm 0.156$ & $1.175 \pm 0.117$ \\
\cline { 2 - 5 } & Boggy soil & $6.375 \pm 0.357$ & $5.695 \pm 0.186$ & $3.715 \pm 0.127$ \\
\cline { 2 - 5 } & Gray cinnamon soil & $1.287 \pm 0.312$ & $1.115 \pm 0.165$ & $0.655 \pm 0.121$ \\
\hline \multirow{4}{*}{\begin{tabular}{c} 
Bird Island Reserve \\
\cline { 2 - 5 }
\end{tabular}} & Carbonate soil & $4.791 \pm 0.213$ & $3.593 \pm 0.128$ & $2.497 \pm 0.139$ \\
\cline { 2 - 5 } & Alpine scrub soil & $6.593 \pm 0.216$ & $4.792 \pm 0.131$ & $3.576 \pm 0.157$ \\
\cline { 2 - 5 } & Goggy soil & $9.972 \pm 0.289$ & $7.373 \pm 0.155$ & $5.121 \pm 0.157$ \\
\hline \multirow{3}{*}{$\begin{array}{c}\text { Upper reaches } \\
\text { of Buha River }\end{array}$} & Carbonate soil & $3.532 \pm 0.212$ & $2.724 \pm 0.135$ & $1.773 \pm 0.115$ \\
\cline { 2 - 5 } & Alpine scrub soil & $3.893 \pm 0.256$ & $1.993 \pm 0.129$ & $1.537 \pm 0.127$ \\
\cline { 2 - 5 } & Boggy soil & $8.772 \pm 0.311$ & $2.592 \pm 0.116$ & $1.976 \pm 0.131$ \\
\cline { 2 - 5 } & Gray cinnamon soil & $2.531 \pm 0.312$ & $1.714 \pm 0.155$ & $4.911 \pm 0.129$ \\
\hline
\end{tabular}

Stipa glareosa, Artemisia anethifolia and Clematis tangutica $\left(F_{15,64}=4.49, p=0.003\right)$. The high-selenium plants were mainly Achnatherum splendens, Carex stenophylla, Stipa glareosa and Artemisia desertorum, among which the selenium content of Achnatherum splendens was the highest, but seasonality was not obvious. The seasonal dynamics of selenium content in different forage varieties were obvious (Artemisia frigida: $F_{2,12}=3.75, p=0.005$; Iris lactea: $F_{2,12}=$ 2.53, $p=0.007$; Carex stenophylla: $F_{2,12}=2.89, p=$ 0.004; Clematis tangutica: $F_{2,12}=2.53, p=0.002$; Stipa purpurei: $F_{2,12}=2.89, p=0.008$; Poa pratensis: $F_{2,12}=3.13, p=0.001 ;$ Elymus nutans: $F_{2,12}=2.22$, $p^{2,}=0.001 ;$ Orinus kokonorica: $F_{2,12}=4.23$, $p=0.008$; Artemisia anethifolia: $F_{2,12}=3.23, p=0.007$; Artemisia desertorum: $F_{2,12}=4.27, p=0.003$; Stellera chamaejasme: $F_{2,12}=3.35, p=0.002$; Achnatherum splendens: $F_{2,12}=4.27, p=0.006$; Agropyron cristatum: $F_{2,12}=2.76, p=0.005 ;$ Thermopsis lanceolata: $F_{2,12}^{2,12}=3.12, p=0.002 ;$ Stipa penicillata: $F_{2,12}=3.56$, $p=0.006$; Stipa glareosa: $F_{2,12}=2.12, p=0.003$ ).

In the Bird Island Reserve, there were significant differences in selenium content among forage varieties. There were very significant differences in selenium content of Artemisia frigida, Artemisia desertorum, Stellera chamaejasme, Achnatherum splendens, Iris lactea, Carex stenophylla, Stipa purpurei, Agropyron cristatum, Poa pratensis, Elymus nutans, Orinus kokonorica, Thermopsis lanceolata, Stipa penicillata, Stipa glareosa, Artemisia anethifolia and Clematis tangutica $\left(F_{15,64}=3.17, p=0.001\right)$. The high-selenium plants were mainly Achnatherum splendens, Carex stenophylla, Stipa glareosa and Artemisia desertorum, among which the selenium content of Achnatherum splendens was the highest, but the seasonality was not obvious. The seasonal dynamics of selenium content in different forage varieties were obvious
(Artemisia frigida: $F_{2,12}=2.89, p=0.000 ;$ Iris lactea: $F_{2,12}=1.73, p=0.000 ;$ Carex stenophylla: $F_{2,12}=2.36$, $p \stackrel{2,12}{=} 0.000$; Clematis tangutica: $F_{2,12}=3.83, \stackrel{p}{p}=0.003$; Stipa purpurei: $F_{2,12}=2.13, p=0.006$; Poa pratensis: $F_{2,12}=2.43, p=0.005$; Elymus nutans: $F_{2,12}=2.83$, $p^{2,12}=0.003 ;$ Orinus kokonorica: $F_{2,12}=2.03$, $p=0.009 ;$ Artemisia anethifolia: $F_{2,12}=2.77, p=0.001$; Artemisia desertorum: $F_{2,12}=2.33, p=0.001$; Stellera chamaejasme: $F_{2,12}=1.82, p=0.002 ;$ Achnatherum splendens: $F_{2,12}=2.24, p=0.008$; Agropyron cristatum: $F_{2,12}=2.65, p=0.001 ;$ Thermopsis lanceolata: $F_{2,12}=2.76, p=0.005$; Stipa penicillata: $F_{2,12}=2.73$, $p=0.002$; Stipa glareosa: $F_{2,12}=3.17, p=0.001$ ).

In the upper reaches of the Buha River area, there were significant differences in selenium content of different forage varieties. There were very significant differences in selenium content of Artemisia frigida, Artemisia desertorum, Stellera chamaejasme, Achnatherum splendens, Iris lactea, Carex stenophylla, Stipa purpurei, Agropyron cristatum, Poa pratensis, Elymus nutans, Orinus kokonorica, Thermopsis lanceolata, Stipa penicillata, Stipa glareosa, Artemisia anethifolia and Clematis tangutica $\left(F_{15,64}=5.23\right.$, $p=0.002)$. The main high-selenium plants included Achnatherum splendens, Carex stenophylla, Stipa glareosa and Artemisia desertorum, among which selenium content of Achnatherum splendens was highest, but the seasonality was not obvious. The seasonal dynamics of selenium content in different forage varieties was obvious (Artemisia frigida: $F_{2,12}=2.73, p=0.001$; Iris lactea: $F_{2,12}=2.33, p=0.002 ;$ Carex stenophylla: $F_{2.12}=2.77, p=0.001$; Clematis tangutica: $F_{2,12}=3.21, p=0.002 ;$ Stipa purpurei: $F_{2,12}=2.75, \stackrel{p}{p}=0.002$; Poa pratensis: $F_{2,12}=2.67$, $p \stackrel{2,12}{=} 0.002$; Elymus nutans: $F_{2,12}=3.81, \stackrel{p}{p}=0.007$; Orinus kokonorica: $F_{2,12}=2.73, p=0.002$; Artemisia anethifolia: $F_{2,12}=2.91, p=0.006$; Artemisia desertorum: 
Table 4. Seasonal dynamics of selenium content in mixed forage of Przewalski's gazelle habitats $\left(\mu \mathrm{g} \cdot \mathrm{g}^{-1}\right)$.

\begin{tabular}{|c|c|c|c|c|}
\hline Regions & Grassland types & Seedling period & Growing period & Withering period \\
\hline \multirow{8}{*}{ Hudong area } & S. chamaeiasme grassland & $0.019 \pm 0.006$ & $0.014 \pm 0.008$ & $0.018 \pm 0.001$ \\
\hline & A. desertorum scrub & $0.023 \pm 0.005$ & $0.018 \pm 0.003$ & $0.024 \pm 0.004$ \\
\hline & A. frigida grassland & $0.011 \pm 0.003$ & $0.008 \pm 0.005$ & $0.012 \pm 0.004$ \\
\hline & I. lactea grassland & $0.029 \pm 0.007$ & $0.023 \pm 0.006$ & $0.027 \pm 0.006$ \\
\hline & A. splendens grassland & $0.037 \pm 0.006$ & $0.031 \pm 0.005$ & $0.039 \pm 0.003$ \\
\hline & S. penicillata grassland & $0.022 \pm 0.003$ & $0.011 \pm 0.003$ & $0.021 \pm 0.003$ \\
\hline & S. glareosa grassland & $0.032 \pm 0.012$ & $0.027 \pm 0.002$ & $0.031 \pm 0.002$ \\
\hline & S. purpurei grassland & $0.015 \pm 0.002$ & $0.012 \pm 0.001$ & $0.015 \pm 0.007$ \\
\hline \multirow{8}{*}{ Bird Island Reserve } & S. chamaeiasme grassland & $0.237 \pm 0.051$ & $0.132 \pm 0.022$ & $0.219 \pm 0.028$ \\
\hline & A. desertorum scrub & $0.313 \pm 0.042$ & $0.155 \pm 0.031$ & $0.264 \pm 0.039$ \\
\hline & A. frigida grassland & $0.244 \pm 0.053$ & $0.123 \pm 0.021$ & $0.193 \pm 0.046$ \\
\hline & I. lactea grassland & $0.211 \pm 0.035$ & $0.132 \pm 0.032$ & $0.184 \pm 0.057$ \\
\hline & A. splendens grassland & $0.557 \pm 0.042$ & $0.149 \pm 0.031$ & $0.319 \pm 0.037$ \\
\hline & S. penicillata grassland & $0.151 \pm 0.036$ & $0.083 \pm 0.042$ & $0.126 \pm 0.056$ \\
\hline & S. glareosa grassland & $0.287 \pm 0.023$ & $0.165 \pm 0.023$ & $0.231 \pm 0.068$ \\
\hline & S. purpurei grassland & $0.175 \pm 0.061$ & $0.103 \pm 0.051$ & $0.135 \pm 0.057$ \\
\hline \multirow{8}{*}{$\begin{array}{l}\text { Upper reaches } \\
\text { of Buha River }\end{array}$} & S. chamaeiasme grassland & $0.067 \pm 0.0075$ & $0.031 \pm 0.0014$ & $0.049 \pm 0.0017$ \\
\hline & A. desertorum scrub & $0.043 \pm 0.0052$ & $0.025 \pm 0.0028$ & $0.034 \pm 0.0034$ \\
\hline & A. frigida grassland & $0.024 \pm 0.0034$ & $0.011 \pm 0.0022$ & $0.019 \pm 0.0016$ \\
\hline & I. lactea grassland & $0.111 \pm 0.0093$ & $0.007 \pm 0.0032$ & $0.009 \pm 0.0023$ \\
\hline & A. splendens grassland & $0.137 \pm 0.0064$ & $0.009 \pm 0.0012$ & $0.119 \pm 0.0022$ \\
\hline & S. penicillata grassland & $0.021 \pm 0.0096$ & $0.013 \pm 0.0031$ & $0.024 \pm 0.0012$ \\
\hline & S. glareosa grassland & $0.067 \pm 0.0075$ & $0.031 \pm 0.0014$ & $0.049 \pm 0.0017$ \\
\hline & S. purpurei grassland & $0.043 \pm 0.0052$ & $0.025 \pm 0.0028$ & $0.034 \pm 0.0034$ \\
\hline
\end{tabular}

$F_{2,12}=3.33, \quad p=0.002 ;$ Stellera chamaejasme: $F_{2,12}=4.12, p=0.009 ;$ Achnatherum splendens: $F_{2,12}=3.24$, $p=0.003 ;$ Agropyron cristatum: $F_{2,12}=3.17, p=0.002$; Thermopsis lanceolata: $F_{2,12}=3.12, p=0.003$; Stipa penicillata: $F_{2,12}=3.72, \stackrel{p}{p}=0.003 ;$ Stipa glareosa: $\left.F_{2,12}=3.23, p \stackrel{2,12}{=} 0.002\right)$.

\section{Discussion and Conclusion}

\section{Selenium Nutrition of Soil}

\section{Total Selenium Content in Soil}

Organisms cannot synthesize and store selenium, and the selenium needed for life must come from soil and forage [10]. When soil has total selenium content $<0.04 \mu \mathrm{g} \cdot \mathrm{g}^{-1}$, it is considered to be severely selenium deficient; total selenium content in soil of $0.04-0.08 \mu \mathrm{g} \cdot \mathrm{g}^{-1}$ is the minimum requirement of selenium; and total selenium content in soil $>0.08 \mu \mathrm{g} \cdot \mathrm{g}^{-1}$ is an adequate requirement of selenium [9, 11, 12]. According to the above criteria, surface soil in the Hudong area was seriously deficient in selenium, was marginally selenium deficient in the upper reaches of the Buha River, and that of Bird Island Reserve should meet the needs of animals and human beings. The selenium content of soil in different regions all showed surface soil accumulation, and decreased with soil depth: the selenium content in surface soil was highest, followed by subsurface soil and substratum soil. Soil type also had a significant effect on selenium content; the selenium content of boggy soil was the highest, and its selenium content in Hudong, Bird Island Reserve and upper reaches of the Buha River should meet the needs of animals and human beings. The selenium content was the lowest in gray cinnamon soil, with only Bird Island Reserve barely reaching minimum requirements, and for Hudong and upper reaches of the Buha River it was significantly lower than $0.04 \mu \mathrm{g} \cdot \mathrm{g}^{-1}$. Therefore, there 
Table 5. Seasonal dynamics of selenium content in different forage varieties of Przewalski's gazelle habitats $\left(\mu \mathrm{g} \cdot \mathrm{g}^{-1}\right)$.

\begin{tabular}{|c|c|c|c|c|}
\hline Regions & Forage varieties & Seedling stage & Growing period & Withering period \\
\hline \multirow{16}{*}{ Hudong area } & A. frigida & $0.0133 \pm 0.0052$ & $0.0143 \pm 0.0025$ & $0.0217 \pm 0.0063$ \\
\hline & I. lactea & $0.0337 \pm 0.0043$ & $0.0227 \pm 0.0041$ & $0.0343 \pm 0.0073$ \\
\hline & C. stenophylla & $0.0457 \pm 0.0051$ & $0.0248 \pm 0.0033$ & $0.0467 \pm 0.0065$ \\
\hline & C. tangutica & $0.0624 \pm 0.0032$ & $0.0524 \pm 0.0043$ & $0.0637 \pm 0.0039$ \\
\hline & S. purpurei & $0.0351 \pm 0.0023$ & $0.0272 \pm 0.0037$ & $0.0399 \pm 0.0031$ \\
\hline & P. pratensis & $0.0157 \pm 0.0015$ & $0.0113 \pm 0.0028$ & $0.0153 \pm 0.0043$ \\
\hline & E. nutans & $0.0268 \pm 0.0023$ & $0.0213 \pm 0.0037$ & $0.0267 \pm 0.0037$ \\
\hline & O. kokonorica & $0.0523 \pm 0.0042$ & $0.0545 \pm 0.0047$ & $0.0527 \pm 0.0029$ \\
\hline & A. anethifolia & $0.0273 \pm 0.0027$ & $0.0137 \pm 0.0012$ & $0.0113 \pm 0.0029$ \\
\hline & A. desertorum & $0.0577 \pm 0.0052$ & $0.0558 \pm 0.0067$ & $0.0558 \pm 0.0035$ \\
\hline & S. chamaejasme & $0.0351 \pm 0.0027$ & $0.0216 \pm 0.0039$ & $0.0297 \pm 0.0041$ \\
\hline & A. plendens & $0.0765 \pm 0.0042$ & $0.0777 \pm 0.0053$ & $0.0771 \pm 0.0029$ \\
\hline & A. cristatum & $0.0332 \pm 0.0021$ & $0.0214 \pm 0.0046$ & $0.0316 \pm 0.0033$ \\
\hline & T. lanceolata & $0.0121 \pm 0.0013$ & $0.0101 \pm 0.0037$ & $0.0127 \pm 0.0037$ \\
\hline & S. penicillata & $0.0273 \pm 0.0021$ & $0.0172 \pm 0.0011$ & $0.0253 \pm 0.0013$ \\
\hline & S. glareosa & $0.0579 \pm 0.0045$ & $0.0323 \pm 0.0024$ & $0.0575 \pm 0.0017$ \\
\hline \multirow{16}{*}{ Bird Island Reserve } & A. frigida & $0.0232 \pm 0.0051$ & $0.0213 \pm 0.0025$ & $0.0217 \pm 0.0023$ \\
\hline & I. lactea & $0.0395 \pm 0.0062$ & $0.0125 \pm 0.0041$ & $0.0413 \pm 0.0073$ \\
\hline & C. stenophylla & $0.0493 \pm 0.0075$ & $0.0349 \pm 0.0033$ & $0.0497 \pm 0.0065$ \\
\hline & C. tangutica & $0.0737 \pm 0.0027$ & $0.0525 \pm 0.0043$ & $0.0557 \pm 0.0039$ \\
\hline & S. purpurei & $0.0378 \pm 0.0017$ & $0.0312 \pm 0.0037$ & $0.0399 \pm 0.0031$ \\
\hline & p. pratensis & $0.0196 \pm 0.0013$ & $0.0177 \pm 0.0028$ & $0.0193 \pm 0.0043$ \\
\hline & E. nutans & $0.0265 \pm 0.0017$ & $0.0113 \pm 0.0037$ & $0.0267 \pm 0.0037$ \\
\hline & O. kokonorica & $0.0575 \pm 0.0025$ & $0.0373 \pm 0.0047$ & $0.0527 \pm 0.0029$ \\
\hline & A. anethifolia & $0.0389 \pm 0.0015$ & $0.0297 \pm 0.0041$ & $0.0373 \pm 0.0029$ \\
\hline & A. desertorum & $0.0597 \pm 0.0041$ & $0.0355 \pm 0.0067$ & $0.0548 \pm 0.0035$ \\
\hline & S. chamaejasme & $0.0448 \pm 0.0025$ & $0.0315 \pm 0.0039$ & $0.0497 \pm 0.0041$ \\
\hline & A. plendens & $0.0873 \pm 0.0037$ & $0.0836 \pm 0.0053$ & $0.0811 \pm 0.0029$ \\
\hline & A. cristatum & $0.0319 \pm 0.0052$ & $0.0273 \pm 0.0046$ & $0.0316 \pm 0.0033$ \\
\hline & T. lanceolata & $0.0173 \pm 0.0028$ & $0.0148 \pm 0.0031$ & $0.0182 \pm 0.0027$ \\
\hline & S. penicillata & $0.0233 \pm 0.0021$ & $0.0173 \pm 0.0012$ & $0.0235 \pm 0.0013$ \\
\hline & S. glareosa & $0.0656 \pm 0.0075$ & $0.0421 \pm 0.0024$ & $0.0659 \pm 0.0012$ \\
\hline \multirow{7}{*}{$\begin{array}{l}\text { Upper reaches } \\
\text { of Buha River }\end{array}$} & A. frigida & $0.0157 \pm 0.0011$ & $0.0163 \pm 0.0021$ & $0.0162 \pm 0.0014$ \\
\hline & I. lactea & $0.0485 \pm 0.0031$ & $0.0327 \pm 0.0041$ & $0.0453 \pm 0.0042$ \\
\hline & C. stenophylla & $0.0453 \pm 0.0042$ & $0.0348 \pm 0.0033$ & $0.0477 \pm 0.0045$ \\
\hline & C. tangutica & $0.0717 \pm 0.0051$ & $0.0524 \pm 0.0043$ & $0.0713 \pm 0.0039$ \\
\hline & S. purpurei & $0.0728 \pm 0.0032$ & $0.0621 \pm 0.0037$ & $0.0799 \pm 0.0053$ \\
\hline & P. pratensis & $0.0176 \pm 0.0023$ & $0.0157 \pm 0.0031$ & $0.0183 \pm 0.0033$ \\
\hline & E. nutans & $0.0365 \pm 0.0017$ & $0.0213 \pm 0.0037$ & $0.0367 \pm 0.0037$ \\
\hline
\end{tabular}


Table 5. Continued.

\begin{tabular}{|c|c|c|c|c|}
\hline \multirow{9}{*}{$\begin{array}{c}\text { Upper reaches of Buha } \\
\text { River }\end{array}$} & O. kokonorica & $0.0525 \pm 0.0025$ & $0.0632 \pm 0.0033$ & $0.0437 \pm 0.0032$ \\
\hline & A. anethifolia & $0.0279 \pm 0.0015$ & $0.0137 \pm 0.0011$ & $0.0273 \pm 0.0019$ \\
\hline & A. desertorum & $0.0587 \pm 0.0031$ & $0.0555 \pm 0.0047$ & $0.0558 \pm 0.0045$ \\
\hline & S. chamaejasme & $0.0338 \pm 0.0025$ & $0.0311 \pm 0.0019$ & $0.0297 \pm 0.0021$ \\
\hline & A. plendens & $0.0773 \pm 0.0062$ & $0.0736 \pm 0.0051$ & $0.0772 \pm 0.0021$ \\
\hline & A. cristatum & $0.0229 \pm 0.0052$ & $0.0231 \pm 0.0036$ & $0.0226 \pm 0.0031$ \\
\hline & T. lanceolata & $0.0149 \pm 0.0028$ & $0.0118 \pm 0.0037$ & $0.0139 \pm 0.0037$ \\
\hline & S.penicillata & $0.0299 \pm 0.0021$ & $0.0193 \pm 0.0012$ & $0.0297 \pm 0.0013$ \\
\hline & S. glareosa & $0.0613 \pm 0.0073$ & $0.0323 \pm 0.0021$ & $0.0597 \pm 0.0013$ \\
\hline
\end{tabular}

were significant differences in soil selenium content among regions and soil types.

\section{Content of Available Selenium in Soil}

The total selenium in soil indicates the selenium capacity in soil, but does not reflect the level of selenium supplied by soil to plants [9-12]. The form of selenium in soil determines its availability, and the available selenium in plants includes selenate, selenite and organic selenium [11]. In our study, the great differences in extraction ability of different extractants and the lack of a uniform extraction method meant that it was difficult to define a critical value for available selenium deficiency [12-13]. Therefore, most studies have focused on factors affecting selenium availability in soil. The content of available selenium mainly depends on the total selenium content in soil, but soil $\mathrm{pH}$, redox potential and organic matter are also major factors affecting selenium availability [15-16]. In the soil types that we studied, the boggy soil had $\mathrm{pH}$ 7.8-8.1, alpine scrub soil had $\mathrm{pH}$ 7.3-7.8, carbonate soil had $\mathrm{pH}$ 7.8-8.3 and gray cinnamon soil had $\mathrm{pH}$ 7.1-8.1. In alkaline soil, selenium mainly exists as selenate and is easily dissolved in water, whereas in neutral or subacid soil, selenium mainly exists as selenite, and is easily bound to iron in soil and forms insoluble complexes that are not easily absorbed by plants [17-18]. The redox state of soil changes selenium availability by affecting its valence state: selenium is the most stable and is difficult for plants to absorb under reducing conditions, and selenium is the most unstable and availability is clearly higher in an oxidizing state [19-20]. Soil organic matter can fix available selenium in soil, but mineralization of organic matter releases a large amount of selenium and increases the available selenium in soil [22-23]. Therefore, there were significant differences in available selenium content among the different soil types in Przewalski's gazelle habitats, which would affect the selenium content in plants and the distribution of selenium in pastures.

\section{Selenium Nutrition of Forage}

Selenium is a rare element with active chemical properties and has a low content in the ecosystem; both deficiency and excess of selenium affect animal and human health [23-24]. In the biogeochemical cycle, soil is the main source of selenium and can meet the needs for selenium of animals and human beings via plants. Selenium is not required for plant growth and development, but selenium content in plants has an irreplaceable role in animal and human health [25-26]. The selenium content in forage is mainly affected by the selenium content in soil, but soil properties, climatic conditions, forage species and grassland types have different effects on selenium content of plants [27-28]. In our study, there were significant differences in selenium content among different plant species, with selenium content of Achnatherum splendens the highest and that of Thermopsis lanceolata the lowest; the other high-selenium plants were Clematis tangutica, Orinus kokonorica and Artemisia desertorum. One measure to balance selenium nutrition in pastures that are selenium deficient is to increase the proportion of high-selenium plants and reseed grassland. In the same area, there were significant differences in selenium contents among grassland types: that of the mixed forage in Achnatherum splendens grassland was highest and that of mixed forage of Stipa purpurei grassland was lowest. Other high-selenium pastures included Stellera chamaejasme, Iris lactea and Stipa glareosa grasslands. Expanding the range of feeding and the forage in different pastures are also methods to balance selenium nutrition. In our study, the selenium content in soil and forage in Hudong was obviously lower than the normal value, and would not meet the needs of animal growth and development; notably, selenium deficiency was more serious in the forage growing period. During the green grass stage, the selenium content of mixed forage in Achnatherum splendens grassland was highest and that of mixed forage of Artemisia frigida and Stipa penicillata grasslands were the lowest. Therefore, it 
is necessary to increase selenium nutrition in such pastures by reseeding and fertilization; additionally, establishing large-scale artificial grassland with high selenium content and reducing the number of grazing animals would increase the basic food of Przewalski's gazelle.

\section{Distribution Characteristics of Selenium Nutrition and its Balance Measures}

\section{Distribution Characteristics of Selenium Nutrition}

Hudong is an area of serious selenium deficiency, the upper reaches of the Buha River have subclinical selenium deficiency and the pastures of Bird Island Reserve can meet the selenium needs of animals. The selenium in soil showed surface soil accumulation, being highest in surface soil and decreased with soil depth. There were significant differences in selenium content among the grassland types, and seasonality was obvious; the selenium content of mixed forage in Achnatherum splendens grassland was highest and in mixed forage of Stipa purpurei grassland was the lowest. There were also significant differences in selenium content among the forage varieties, and selenium content highest in the seedling stage and lowest in the growing period.

\section{Balance Measures for Selenium Nutrition}

Applying selenium fertilizer, improving soil and reseeding high-selenium plants can effectively increase selenium nutrition in pastures. We found that the selenium content in soil and forage significantly differed among regions; therefore, removing fences, establishing habitat corridors, expanding habitat and increasing feeding areas could all be beneficial for the selenium balance of Przewalski's gazelle. However, the effects of feeding and nomadic behaviors of Przewalski's gazelle on selenium nutrition needs further study.

\section{Acknowledgements}

We acknowledge support by the Program of the National Natural Science Foundation of China (No. 41671041), the Project of National Key Research and Development Program of China in the 13th Five-year Plan (2016YFC0502601), and the Southwest University of Science and Technology Doctoral Research Fund (No. 17zx7146).

\section{Conflict of Interest}

The authors declare no conflict of interest.

\section{References}

1. LI Z.K. Analysis of Se concentrations in study farm of Sanjiaocheng in Qinghai at different seasons. Journal of Traditional Chinese Veterinary Medicine, 7, 45, 2009.

2. SHEN X.Y. Forage strategy of Przewalski's gazelle under selenium stress. Acta Ecologica Sinica, 29 (6), 277, 2009.

3. CHAN Y.H., SIU C.W., YIU K.H., YIU K. H., CHAN H. T., LI S. W., TAM S., CHEUNG B.M. Adverse systemic arterial function in patients with selenium deficiency. Journal of Nutrition Health \& Aging, 16 (1), 85, 2012.

4. NANGLIYA V., SHARMA A., YADAV D., SUNDER S., NIJHAWAN S., MISHRA S. Study of trace elements in liver cirrhosis patients and their role in prognosis of disease. Biological Trace Element Research, 165 (1), 35, 2015.

5. MICKIEWICZ B., VILLEMAIRE M.L., SANDERCOCK L.E., JIRIK F.R., VOGEL, H.J. Metabolic changes associated with selenium deficiency in mice. Biometals, 27 (6), 1137, 2014.

6. HURST R., SIYAME E.W.P., YOUNG S.D., CHILIMBA A.D.C., JOY E.J.M., BLACK C.R. Soil-type influences human selenium status and underlies widespread selenium deficiency risks in malawi. Scientific Reports, 3 (1425), 1425, 2013.

7. XU S.W., YAO H.D., ZHANG J., ZHANG Z.W., WANG J.T., ZHANG J.L. The oxidative damage and disbalance of calcium homeostasis in brain of chicken induced by selenium deficiency. Biological Trace Element Research, 151 (2), 225, 2013.

8. ZHANG J.L., ZHANG Z.W., SHAN A.S., XU S.W. Effects of dietary selenium deficiency or excess on gene expression of selenoprotein $\mathrm{n}$ in chicken muscle tissues. Biological Trace Element Research, 157 (3), 234, 2014.

9. SHEN X.Y, LI X., ZHANG R.D. Studies of "unsteady gait disease" of the Tibetan gazell. Journal of Wildlife Diseases, 46 (2), 560, 2013.

10. SUTTLE N.F. Mineral nutrition of livestock ( $4^{\text {th }}$ edition). London: CABI publishing, 587, 2013.

11. SHEN X.Y., CHI Y.K., HUO B., WU T., XIONG K.N. Effect of fertilization on ryegrass quality and mineral metabolism in grazing the Wumeng semi-fine wool sheep $[\mathrm{J}]$. Fresenius Environmental Bulletin 27(10), 6824, 2018.

12. ZHANG Q, JIANG Z.G., WANG C., DAVID M. Impacts of grassland fence on the behavior and habitat area of the critically endangered przewalski's gazelle around the Qinghai lake. Science Bulletin, 58 (18), 2262, 2013.

13. LI J.F., NIE Z.J., ZHAO P., GAO W., LIU H.E. Research progress on selenium nutrition in the soil-plant system. Journal of Southern Agriculture, 47, 649, 2016.

14. CHI Y.K., XIONG K.N., CHEN H., MIN X.Y., LIAO J.J., SHEN X.Y. Effect of grazing to copper pollution meadow on copper metabolism in Wumeng semi-fine wool sheep. Polish Journal of Environmental Studies 28(3), 1, 2019. DOI: $10.15244 /$ pjoes/87102

15. YANG J.Q., LUO Q.J., YANG K.L., ZHANG G.Q., WANG X., XIE P.G., ZHANG J.R. Effects of supplementing complex trace elements on the digestion and metabolism of grazing sheep in Yili summer pasture. Journal of Xinjiang Agricultural University, 38, 173, 2015.

16. SUN H.J., RATHINASABAPATHI B., WU B., LUO J., PU L. P., MA L.Q. Arsenic and selenium toxicity and their interactive effects in humans. Environment International, 69, 148, 2014. 
17. SHEN X. Y., JIANG Z.G. Studies of dynamics of content of trace elements in herbage on shouqu grassland in yellow river. Chinese Journal of Grassland, 30, 120, 2008.

18. JIANG Y., ZENG Z.H., YANG Q.S., ZHAO J., YANG Y.D., HU, Y.G. selenium (se)uptake and transformation mechanisms and physiological function in plant: a review. Chinese Journal of Applied Ecology, 27 (12), 4067, 2016.

19. WANG X.F., CHEN S.Y., LUO Z., HUANG Q.Q., QIAO Y.H., SU, H.J., LI HF. Mechanisms of selenium uptake, translocation and chemical speciation transformation in plants. Journal of Agricultural Resources \& Environment, 31, 539, 2014.

20. CHI Y.K., XIONG K.N., ZHANG Z.Z. Research on photosynthetic interannual dynamics of gramineous forage in the karst rocky desertification regions of South China. Oxidation Communications, 39 (3), 2476, 2016.

21. SONG S.Z., XIONG K.N., CHI Y.K., SHEN X.Y., GUO T., LU N.N. Research progress and prospect of grassland establishment and ecological animal husbandry in the karst rocky desertification area. Fresenius Environmental Bulletin 27(10), 7017, 2018.

22. ZHOU L.Y., PU X.Y. Research on the trace element contents of a soil-grass-Przewalski's gazelle ecosystem in Hudong, Qinghai, China. Acta Prataculturae Sinica, 21, 145, 2012.
23. LI J.F., NIE Z.J., ZHAO P., GAO W., LIU H.E. Research progress on selenium nutrition in the soil-plant system. Journal of Southern Agriculture, 47 (5), 649, 2016.

24. LI H.R., YANG L.S., TAN J.A.,WANG W.Y., HOU S.F., LI Y.H. Progress on selenium deficiency in geographical environment and its health impacts in China. Current Biotechnology, 7(5), 381, 2017.

25. HOU S.F. Change trend and factors of residential selenium nutrition status in low selenium belt in China. Geographical Research, 167 (3), 660, 2000.

26. ZHANG J.H., ZHU L.F., YU S.M., JIN Q.Y. Selenium cycling and transformation in paddy field and selenium nutrition of rice: a review. Chinese Journal of Applied Ecology, 23 (10), 2900, 2012.

27. ZHANG J.H., ZHU L.F., YU S.M., JIN Q.Y. Selenium cycling and transformation in paddy field and selenium nutrition of rice: a review. Chinese Journal of Applied Ecology, 23 (10), 2900, 2012.

28. WANG X.H., XIANG Y.Z., SONG S.L., QU F.R., WANG L., GUAN S.F. Relationship between the environment selenium nourishment level and the incidence of Keshan disease. Chinese Journal of Control of Endemic Disease, 11, 35, 2005. 
